\title{
Towards a Proof-Theoretic Semantics of Equalities
}

\author{
Reinhard Kahle
}

\begin{abstract}
We have a fresh look on Frege's mode of presentation, taking into account proofs of equalities as a key concept. Revisiting the classical example of Morning star and Evening star the account leads to a proposal for a proof-theoretic semantics of equalities.
\end{abstract}

Keywords Frege - Mode of presentation - Sinn und Bedeutung - Proof-theoretic semantics $\cdot$ Equality

\section{Frege's Question}

Gottlob Frege opened his seminal paper Sinn und Bedeutung [3] by asking what the epistemic difference is between the equations $a=a$ and $a=b .{ }^{1}$ His proposal to distinguish between sense and denotation (or reference) of a term turned out to be one of the most fruitful conceptual advances in the history of philosophical logic.

Modern Possible Worlds Semantics draws on this distinction: the sense of a term refers to the full variety of possible worlds (in the way that we have to consider the denotation of a term in every possible world), while the (Fregean) denotation has to take into account only the actual world.

As appealing as this view might be, there are (at least) two problems with it. First, it comes with a concept of rigid designators. Second, it is not applicable to mathematics, because mathematical equations hold equally in every possible world.

While many approaches try to attack the problem from a semantic perspective, here we would like to provide a syntactic account, which takes up Frege's original question

\footnotetext{
${ }^{1}$ In fact, he doesn't put this directly as a question, but rather states that " $a=a$ holds $a$ priori and, according to Kant, is to be labeled analytic, while statements of the form $a=b$ often contain very valuable extensions of our knowledge and cannot always be established a priori." [5, p. 157].

R. Kahle $(\varangle)$

Departamento de Matemática, CMA and CENTRIA, FCT, Universidade

Nova de Lisboa, 2829-516 Caparica, Portugal

e-mail: kahle@mat.uc.pt

(C) The Author(s) 2016

T. Piecha and P. Schroeder-Heister (eds.), Advances in Proof-Theoretic Semantics,

Trends in Logic 43, DOI 10.1007/978-3-319-22686-6_9
} 
of the (epistemic ${ }^{2}$ ) difference of $a=a$ and $a=b$. With restriction to singular terms, we will propose a fresh understanding of Frege's mode of presentation. It is motivated by the question how we actually prove a particular equation, and it can be considered as a proof-theoretic semantics of equalities.

\section{Equality Versus Identity}

To set the stage for the further discussion we would like to assume that we always use a first-order language for which we may have some non-logical axioms, and a fixed structure with universe $\mathfrak{A}$ in which this language is interpreted ${ }^{3}$ by an interpretation function $(\cdot)^{\mathfrak{M}}$. Let us use Latin characters for terms of the language, and German (Gothic) ones for elements of the structure. Equality is understood as the relation $t=s$ on the syntactical level between terms of the first-order language ${ }^{4}$; identity stands for the (trivial) relation $\mathfrak{a} \equiv \mathfrak{a}$ on the semantic level, which holds only between an object in the structure and itself. ${ }^{5}$ The fact that identity is not entirely trivial comes from its use for terms in combination with the interpretation in the form $(t)^{\mathfrak{M}} \equiv(s)^{\mathfrak{M} 6}$

In this setting we can recast Frege's first observation that "if we were to regard equality as a relation between that which the names ' $a$ ' and ' $b$ ' designate, it would seem that $a=b$ could not differ from $a=a$ (i.e. provided $a=b$ is true). A relation would thereby be expressed of a thing to itself, and indeed one in which each thing stands to itself but to no other thing." [5, p. 157]. In our terminology we may say that we are not concerned with the semantical identity relation $\mathfrak{a} \equiv \mathfrak{a}$, but with the syntactical equality relation $t=s$.

It is standard to axiomatize equality in first-order logic as a universal congruence relation, i.e., an equivalence relation compatible with all operations (functions and relations). As such, it mimics on the syntactic level just the properties which identity exhibits on the semantic level. But the domain of identity is simply $\mathfrak{A} \times \mathfrak{A}$, and the elements of $\mathfrak{A}$ are unique in the sense that $\mathfrak{a} \equiv \mathfrak{a}$, but $\mathfrak{a} \not \equiv \mathfrak{b}$ for two elements $\mathfrak{a}, \mathfrak{b} \in \mathfrak{A}$. On the syntactic side, however, the equality relation is defined for terms, and, clearly, two terms, though being interpreted by the same object $\mathfrak{a}$, may well be different.

\footnotetext{
${ }^{2}$ In this paper, we restrict ourselves to an epistemic perspective, and we will not go into metaphysical issues. This seems also to be Frege's position, as he speaks explicitly about "our knowledge" (see the citation in the previous footnote).

${ }^{3}$ Here, the notion of structure includes the possibility that its universe is taken from the "real world".

${ }^{4}$ We make here a slight abuse of the word "relation"; strictly speaking, a relation is a semantic concept and "syntactic relation" cannot be anything more than a formula formed by use of a relation symbol. ${ }^{5}$ For a recent philosophical discussion of the concept of identity see also [14].

${ }^{6}$ This distinction of equality and identity may help to unravel Frege's initial footnote in Sense and Denotation explaining that he uses equality "in the sense of identity and understand ' $a=b$ ' in the sense of ' $a$ is the same as $b$ ' or ' $a$ and $b$ coincide'." [5, p. 157, slightly changed translation]; of course, Frege didn't have the modern distinction of syntax and semantics at hand.
} 
Frege introduced the sense of a sign as its mode of presentation. Generally, this is taken as some kind of illustration rather than a definition. It is our aim to provide a more formal explication of mode of presentation by drawing on the difference of equality and identity.

\section{The Mode of Presentation}

Frege did not define the notion of mode of presentation, but he did give two quite illustrating examples of it.

The first one is taken from geometry: a particular point may be presented as intersection of two lines $a$ and $b$ or as intersection of the lines $b$ and $c$. Though the intersections take place at the very same point, we would say that the two modes of presentation differ: one refers to the lines $a$ and $b$, the other to the lines $b$ and $c$.

Assuming a suitable axiom system for geometry, this system will provide terms which serve as definitions for the intersections expressed by, say, $\operatorname{Intsec}(a, b)$ and Intsec $(b, c)$. Assuming that both terms refer to the same point $\mathfrak{p}$ of the plane, it requires some reasoning in the given axiomatic framework to derive the equality Intsec $(a, b)=\operatorname{Intsec}(b, c)$. This equality is epistemically different from a simple reflexive equality, like Intsec $(a, b)=\operatorname{Intsec}(a, b)$.

We propose to use $\operatorname{Intsec}(a, b)$ to obtain a mode of presentation of $\mathfrak{p}$, and $\operatorname{Intsec}(b, c)$ to obtain another mode of presentation of the same point. ${ }^{7}$ We may say that a term $t$ of our formal language expresses (to use Frege's wording) a mode of presentation if it may be used as a mathematical expression to define a newly introduced constant $A$. We do not say that the term is the mode of presentation, as - with Frege - the latter is surely not a syntactic object (this would be the mode of designation, [5, p. 157]). The way the mode of presentation should be located between the purely syntactical level and the semantical level will be discussed in more detail below. But let us note, that our mode of presentation is clearly different from any form of reference in model-theoretic terms.

Let us now turn to the more prominent example given by Frege. By "morning star", Venus is presented as the $\operatorname{star}^{8}$ visible in the morning, by "evening star" as visible in the evening. Thus, the sense of "morning star" differs from that of "evening star", although both refer to the same object. We may use "the star visible in the morning" and "the star visible in the evening" as the expressions which give us the mode of

\footnotetext{
${ }^{7}$ This view is even better illustrated in the example Frege gives in his Begriffsschrift in the paragraph on identity of content, $[4$, p. 20f]. This paragraph could be used as further support of our account here, yet, Frege, by the time of the Begriffsschrift, didn't bring forward the notions of sense or mode of presentation.

${ }^{8}$ In the discussion of this example, "star" is, of course, to be understood as a folk term.
} 
presentation, using the same argument as above: these expressions may serve as terms $t$ defining a constant $A$ ("morning star", "evening star", or even "Venus"). 9

Thus, we may extend our working definition of mode of presentation given above for mathematical terms to terms in general, saying that a term $t$ may express a mode of presentation if it can be used as definiens in a clause like "Let $A$ be $t$." Later we shall see how proofs enter.

\section{Morning Star Versus Evening Star Revisited}

Frege's example of difference of senses in "morning star" and "evening star" became a classic. It is intuitively clear that there are two different senses, although there is only one reference.

Possible worlds semantics does not cope well with this example. Taking Kripke's [9] famous distinction of rigid and non-rigid (use of) terms into account, one can consider "morning star" and "evening star" as definite descriptions ${ }^{10}$ which should be non-rigid. But "Venus", as a proper name, is supposed to be rigid. Now, however, in the worlds in which "morning star" and "evening star" are supposed to be different, we would have "two copies" of Venus, let's call them Venus $M$ and Venus ${ }_{E}$. Leaving aside the question which of them should be the Venus, the problem is that for these two Veneres the astronomical laws have to fail-otherwise they would coincide again. ${ }^{11}$ Is it really the case that- to understand the difference of the sense of morning star and evening star-we would have to consider worlds with different astronomical laws? In our view, the difference in the sense of morning star and evening star should not depend on the astronomical laws at all-it depends, to go back to Frege, only in the mode of their presentation.

In our account, we would take (appropriate) terms $t_{M}$ and $t_{E}$ representing "morning star" and "evening star" in a sufficiently formalized astronomical theory as definite descriptions which both could serve as defining a planet. It is now a new task to prove the equality $t_{M}=t_{E}$ by use of astronomical laws (together with the empirical astronomical observations which are formalized as statements involving $t_{M}$ and $t_{E}$ ). We may say that the fact that the denotations of $t_{M}$ and $t_{E}$ are equal follows from

\footnotetext{
${ }^{9}$ Of course, Venus should be defined by only one of these expressions-unless it is already known that they coincide (though, it would look quite odd to give two different definitions of one and the same object).

${ }^{10}$ Kripke treats the terms "Phosphorus" and "Hespherus" as proper names; we take here "morning star" and "evening star" as elliptic definite descriptions extendable to "the brightest non-lunar object in the morning/evening sky". For more on rigid designators, see [10].

${ }^{11}$ Consider an alternative world where the astronomical observations of $\operatorname{Venus}_{M}$ coincide with the observations of Venus in the real world. If this alternative world have the same astronomical laws as the real world, $\operatorname{Venus}_{M}$ has to appear at the same position as Venus in our world, i.e., at the place of $\operatorname{Venus}_{E}$, i.e., Venus $M$ and Venus $E$ have to be identical.
} 
the identity of $\left(t_{M}\right)^{\mathfrak{M}} \equiv\left(t_{E}\right)^{\mathfrak{M}} \equiv \mathfrak{V} \mathfrak{e n u s}$ in the real world, while the equality of the modes of description $t_{M}=t_{E}$ follows from the proof in our astronomical theory. The need of performing this proof explains the epistemic difference between identities and equalities. ${ }^{12}$

\section{Equality}

We here consider only equalities between terms, which may refer to mathematical objects or to objects of our real world.

As said, in first-order logic, equality is axiomatized as a universal congruence relation, thus directly linked to extensionality (the congruence axioms include the compatibility with all functions and relations).

Working in an epistemic context, however, one may note that not all (true) equalities might be known by an agent ${ }^{13} \mathscr{A}$. Thus, the equalities known by $\mathscr{A}$ may not be complete with respect to the identities which hold in the intended model of $\mathscr{A}$ 's knowledge. This incompleteness has to be understood with respect to the combination of interpretation and identity as described in Sect.2: for two terms $t$ and $s$, $(t)^{\mathfrak{M}} \equiv(s)^{\mathfrak{M}}$ may hold, but $\mathscr{A}$ doesn't know $t=s$.

The incompleteness can arise from two different sources. On the one hand, an agent may have an "underaxiomatized" representation of the world. On the other hand, agents are not supposed to be logically omniscient, and will miss (fail to know) those equations which they haven't yet proved.

The first case may apply in the morning star/evening star example, when the agent does not know the astronomical laws to derive the fact that both terms refer to the same object. $^{14}$

The second case may apply to the geometric example, if the agent didn't perform the mathematical proof of the equality of the two intersections.

In both cases, the equalities the agent knows are incomplete with respect to the identities which hold in the appropriate model. Now, the equality relation $=$ of $\mathscr{A}$ (considered as the set of equalities known by $\mathscr{A}$ ) may serve to express some intensionality with respect to the outer extensionality, given by $\equiv$ (or all true equalities).

\footnotetext{
${ }^{12} \mathrm{We}$ leave here aside the fact that essentially nobody actually performs this proof, but learns the equality $t_{M}=t_{E}$ in school and, thus, adds it somehow as an axiom to the belief set. But as it should be with everything we learn in school, it should be possible, in principle, to replace our "learned axioms" by actual proofs, if we would study the respective topic in sufficient detail.

${ }^{13}$ The term agent is heavily burdened by its use in Artificial Intelligence. However, because of a lack of alternatives, we use "agent" here in the way as it became recently fashionable in philosophy to designate "something having knowledge".

${ }^{14}$ To satisfy our remark of footnote 12 we may stipulate that this agent also didn't learn this equality in school or elsewhere.
} 
If we analyze $\mathscr{A}$ 's knowledge we should allow the substitution of two terms only if $\mathscr{A}$ 's knowledge comprises the corresponding equality-the underlying identity in the model is irrelevant. With only these identities in mind, we may observe the intensional phenomena in $\mathscr{A}$ 's knowledge.

\section{Equality of Senses}

One of the fundamental challenges for every theory of senses is the notion of equality of senses.

In our setting the notion of sense is naturally relativized to (the knowledge of) an agent $\mathscr{A}$. A very naive attempt would be to introduce a notion of equality of senses relativized to an agent $\mathscr{A}$, identifying the sense expressed by two terms if and only if $\mathscr{A}$ can prove the equality $t=s$. This would allow to separate the denotation from the senses of two terms denoting the same object in cases where $\mathscr{A}$ does not have the proof of the corresponding equality at hand. But it would compromise Frege's original idea, as the senses of "morning star" and "evening star" should clearly stay different even if somebody knows that both denote Venus.

Still, we may obtain an interesting notion of equality of senses if we allow for the closure of the mode of presentation under some equalities. This can be illustrated best by use of the geometric example: we said that $\operatorname{Intsec}(a, b)$ and $\operatorname{Intsec}(b, c)$ should be considered as different modes of presentation of the point $\mathfrak{p}$. It seems to be, however, that Intsec $(a, b)$ and Intsec $(b, a)$ do not give us different modes of presentation of the same point. In technical terms, this means that the mode of presentation is not changed when we invoke the symmetry of the relation Intsec.

It is not our aim to specify concrete criteria concerning which (type of) equations should be taken into account for the equality of senses. In contrast, we think that equality of senses should not only be relativized to an agent (or an agent's knowledge) but that it could also be graduated and that it depends on the chosen axiomatic context.

The rôle of the axiomatic context can be exemplified by the natural numbers: if they are introduced as a commutative semigroup, commutativity is, of course, "build in" and $t+s$ should not have a sense different from that of $s+t$. If, however, the natural numbers are introduced by use of the Peano Axioms, the commutativity of addition requires a rather non-trivial proof by induction, and, in this context, one might say that the sense of $t+s$ differs from the one of $s+t$, as the required recursion over (only) one of the summands to calculate the value may lead to substantially different computations.

This last example shows that, for our notion of mode of presentation, the underlying axiomatic setting forms an integral part of the sense of a term. ${ }^{15}$

\footnotetext{
${ }^{15}$ To elaborate this approach one could take into account, for instance, background knowledge as constitutive for senses. We may also invoke definitional knowledge obtained by definitional reflection, [12, Sect. 2.3.2].
} 


\section{Proof-Theoretic Semantics}

So far, we gave some kind of answer to what we called Frege's questions stressing the epistemic character of a possibly incomplete set of proven equalities of an agent, in contrast to identity in a model. We will now turn to the idea of proof-theoretic semantics.

According to ([8], p. 503),

[p]roof-theoretic semantics [assigns] proofs or deductions an autonomous semantic role from the very onset, rather than explaining this role in terms of truth transmission. In prooftheoretic semantics, proofs are not merely treated as syntactic objects [...], but as entities in terms of which meaning and logical consequence can be explained.

This approach is already quite successfully pursued for the usual logical operations (see $[7,12]$ and this volume). It is our aim to extend it to some further concepts, like equalities here or necessity in [6].

In the case of equality a proof-theoretic semantics requires that, from the very onset, one would have to dispense with any (model-theoretic) notion of identity. From a technical point of view, one could say that the proof-theoretic semantics of the equality relation is given by the axioms involving this relation. But what would be the proof-theoretic semantics of a particular equation? The terms in such an equation have now, where any model-theoretic interpretation is gone, of course, an autonomous status.

From a proof-theoretic perspective, "morning star" and "evening star" should, of course, be different. Their mode of presentation is given by the way the axioms introduce them as terms. This includes implicitly the full axiomatic framework which now makes part of the mode of presentation.

Whatever the concrete axioms might be, they should state that the "morning star" is visible (on some days) in the morning, and the "evening star" in the evening, respectively. As discussed above, the equality between them needs a proof. For the proof-theoretic semantics of the terms it should not even be relevant whether such a proof is performed or not-its sheer need gives rise to consider the proof-theoretic semantics as different for the two terms, determined only by the axioms governing them. Only in the case of "immediate" ("trivial" or maybe "elementary") equalitieslike in the case of the symmetry of Intsec - a term might be manipulated without changing its sense.

As related approaches we would like to mention here Tichý's Transparent Intensional Logic (TIL), [1, 13] and Moschovakis's Sense and Denotation as Algorithm and Value, [11].

TIL does not dispense with possible worlds, but assigns them a secondary rôle in the analysis of senses. These are introduced as abstract procedures, called constructions, which are applied to an object, in dependence of a possible world, to decide whether this object (for instance, Venus) fulfills the intension (e.g., being the morning star). With respect to our approach one can ask whether, and if so, in which way the abstract procedures can be related to the proofs we take as a basis. 
Such a relation would be given, at least partly, by the Curry-Howard correspondence for Moschovakis's approach. He introduces senses as algorithms which compute (denotational) values. Based on the well-known correspondence of algorithms and proofs, we could adapt Moschovakis's slogan by describing our (broader) approach to intensionality as Sense and Denotation as Proof and Truth. Conversely, Moschovakis's account could also be dubbed a recursion-theoretic semantics.

Acknowledgments Research supported by the Portuguese Science Foundation, FCT, through the projects Hilbert's Legacy in the Philosophy of Mathematics, PTDC/FIL-FCI/109991/2009; The Notion of Mathematical Proof, PTDC/MHC-FIL/5363/2012; Hilbert's 24th Problem, PTDC/MHCFIL/2583/2014, and UID/MAT/00297/2013.

Open Access This chapter is distributed under the terms of the Creative Commons Attribution Noncommercial License, which permits any noncommercial use, distribution, and reproduction in any medium, provided the original author(s) and source are credited.

\section{References}

1. Duží, M., Jespersen, B., Materna, P.: Procedural Semantics for Hyperintensional Logic. Logic, Epistemology, and the Unity of Science, vol. 17. Springer, Berlin (2010)

2. Frege, G.: Begriffsschrift, Nebert, Halle a.d.s. (English translation [4])

3. Frege, G.: Über Sinn und Bedeutung. Zeitschrift für Philosophie und philosophische Kritik, NF 100 pp. 25-50 (1892) (English translation [5])

4. Frege, G.: Concept script. In: van Heijenoort, J. (ed.) From Frege to Gödel, Harvard University Press, Cambridge (1967) (English translation of [2])

5. Frege, G.: On sense and meaning. In: McGuiness, B. (ed.) Collected Papers on Mathematics, Logic, and Philosophy, p. 157-177. Basil Blackwell (1974) (English translation of [3] by M. Black)

6. Kahle, R.: A proof-theoretic view of necessity. Synthese 148(3), 659-673 (2006)

7. Kahle, R., Schroeder-Heister, P. (eds.) Proof-Theoretic Semantics. Springer (2006). Special issue of Synthese 148(3), 503-743

8. Kahle, R., Schroeder-Heister, P.: Proof-theoretic semantics-introduction. Synthese 148(3), 503506 (2006)

9. Kripke, S.: Naming and Necessity. Harvard University Press, Cambridge (1980)

10. LaPorte, J.: Rigid Designators. In: Zalta, E.N. (ed.) The Stanford Encyclopedia of Philosophy (Summer 2011 edn.) (2011), http://plato.stanford.edu/archives/sum2011/entries/rigiddesignators/

11. Moschovakis, Y.: Sense and denotation as algorithm and value. In: Väänänen, J., Oikkonen, J. (eds.) Logic Colloquium '90, pp. 210-249. Lecture Notes in Logic, vol. 2, Association for Symbolic Logic (1994)

12. Schroeder-Heister, P.: Proof-theoretic semantics. In: Zalta, E.N. (ed.) The Stanford Encyclopedia of Philosophy (Winter 2012 edn.) (2012), http://plato.standard.edu/archives/win2012/ enties/proof-theoretic-semantic/

13. Tichý, P.: The Foundations of Frege's Logic. De Gruyter, Berlin (1988)

14. Wehmeier, K.F.: How to live without identity-and why. Australas. J. Philos. 90(4), 761-777 (2012) 